\title{
FOMs of Consciousness Measurement
}

\author{
W.S. Li \\ Department of Microelectronics, \\ Soochow University \\ China
}

\author{
Y.T. Li \\ Tongda College \\ University of Posts \& Telecommunications \\ China
}

\begin{abstract}
Through engineering eyeglass, the consciousness is on object-oriented knowing and controlling mostly embedded in human brain. If an outstanding expert can invent key tool in single index termed figure of merit (FOM) for representing different levels of consciousness tested, then her/his God's image should attract all eyes of doctors fighting in edge line. And not through general voiced speech this expert can imagine "thanking clock of life for the advances of tiny-sensor-techs" due to silent communication featured no noises disturbing her/his information sink. Here I start with showing triple FOMs for the evaluation of typical test cases of consciousness, and then review the rules in spider charts by scanning main sensors of silent speech interfaces. At last, my auricular point's NIRS-based Broca's area decoding with merits of millisecond resolution is reported. All in all the works built up novel milestones of the first autor proposed brain health microelectronics (BHM).
\end{abstract}

Keywords-consciousness measurement; figure of merits; silent speech interfaces; laws extracting; Broca's area decoding

\section{INTRODUCTION}

The human brain is the ocean of electrochemistry in tandem of digital to analog converter (DAC) and ADC in single direction valve of synapse at more than numbers of light-year scale [1,2].

In August 1998 at Guanqian Book City in Suzhou City, China, one translated science fiction titled The Truth Machine published in 1996 in USA written by Harvard Univ. graduate attracted our eyes, in reason of idea that imagined the fMRI as lie detector! This idea called me to invite a co-worker, doctoral student Mr. Shihua Huang to finish firstly lie test between the eyebrows near the outside of front lobe sensing spontaneous short and middle infrared radiations, in November 2001 at Fudan Univ., as Visiting Scholar of Microelectronics[3].

This successful primer test design case inspired me to invite two co-workers, Professor Liang Guo and doctor Jianhuan Chen, finish the first fMRI based lie detection in November 2002 before the Nobel Prize year 2003 of fMRI at The Affiliated Hospital of Soochow Univ. in mainland China. The above two experiments delayed half a year less than their USA editions dreaming of "Truth shall let you free", one review paper from distinguished Professor L. A Geddes at Purdue Univ. impressed me most with two academic warm reply letters in 2002 and 2004. In February 2005, I pursuited doctoral degree on brain neurotransmitters non-invasive measurement based SOC design. Where is the intelligent hometown? The first answer is human brain. What is the most important thing in your life? Asked known Microelectronics Professor S. M. Sze in Oct. 2002 in Soochow Univ. The only right keyword HEALTH comes from the co-inventor of nonvolatile memory in Jan. 1967 in Bell Labs. So far an older Master student and senior associate Professor, Mr. Tiny Stone, elder son of a kind doctor and Chinese bestselling author, $\mathrm{Mr}$. Wenshi Li was pushed to open the window of BHM focused on human brain information analyses and their microchips design for healthy goals, with my young co-worker, dear Yaotian [3].

The core challenge encounters in top scientist's world as how to measure and model levels of consciousness with the tiny system on chip? The responding applied cycle problems include such imagined words or thinking decoding and willingness controlling as for people her/him self and for other friends.

Deeping into literatures we invent and dig out three kinds of figure of merits (FOMs) for analyses of levels of consciousness (in S2), followed by the illustration of silent communication BCI evolution rules in 6-axes spider chart facing 8-sensors cases (S3), in brief in S4 our auricular point's NIRS-based BCI results of imagined Chinese vowel phonemes decoding are reported.

\section{FOMS OF LEVELS OF CONSCIOUSNESS}

To build apt and valid FOM is an academic paradigm in scientific and technic fields such as golden ratio and resistivity. To push forward further brain health microelectronics we like to introduce three basic FOM models as followed [4].

\section{A. IDEA Law}

How can you win the success? Yes by SYNTHESIS, said the author's peers, in 2007, Kasparov, the former world chess champion. One interesting ideal assessment model of idea was born here as we knew that: IQ (Intelligence Quotient, W. Stern, 1912), EQ (Emotional Quotient, W. L. Payne, 1985) and AQ (Adversity Quotient, P. G. Stoltz, 1997) [Stoltz P. G. Adversity Quotient: Turning Obstacles into Opportunities (Wiley, New York, 1997)]. Now we defined that: DQ is as doctor quotient meaning focused synthesis attitude on doing with three hearts of Ambition, Kindness and Perseverance. To set the maximal values as: (1) $\mathrm{DQ}=\mathrm{A}+\mathrm{K}+\mathrm{P}=1+2+4=7$ and (2) $\mathrm{DQ}=\mathrm{IQ}+\mathrm{EQ}+\mathrm{AQ}=1+2+4=7$, Then had the IDEA law as (3) $\mathrm{IDEA}=(\mathrm{I}+\mathrm{E}) /(\mathrm{D}-\mathrm{A})=(\mathrm{I}+\mathrm{E}) /(\mathrm{I}+\mathrm{E}) \equiv 1$, wherein $\mathrm{ONE}$ represents success. But while the IQ, EQ, and AQ and DQ leap up and down randomly between zero and their Maxes, then through running MATLAB 7.0 program we can illustrate six results in Figure I . Into 6-sub-figures the nests may catch freedom golden ratios for every quotients inputs but touch pity the lower IDEA-peak outputs. In brief, IDEA may be the most 
important single indicator describing the sense of success for every champion of themselves lives.

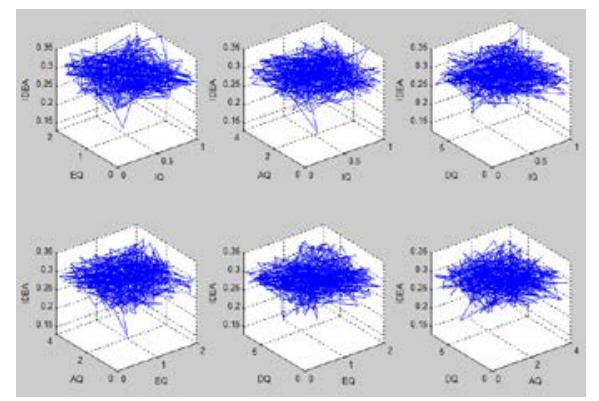

FIGURE I. CHAOTIC IQ, EQ, AND AQ AND DQ NOT EMBRACE LARGER IDEA INDEXES ONLY IF WITH BALANCED VALUES AND PROPORTIONS IN 4Q JUST LIKE KASPAROV ONCE EMPHASIZED IN HIS BOOK IN 2007 COMPARED WITH TRUTH-Q VERSUS LIE-Q AND THEIR COMPLEMENTARY LIE-TRUTH LAW IN FIRST AUTHOR'S DOCTORAL DISSERTATION IN 2005 2009.

\section{B. Index of Consciousness}

In 2013, Prof. M. Massimini at Univ. of Mian led his team to develop a tool called perturbational complexity index (PCI, ranging $0 \sim 1$ ) for consciousness assessment in research paradigm of TMS+High-Density-EEG+PCI [5]. This found index tool was used to identify the integrated information values after subjects (32 of healthy ones and 20 of patients) stimulated by TMS. They reported results such as PCI= $0.44 \sim 0.67$ for healthy people during wakefulness while PCI= $0.51 \sim 0.62$ for locked-in patients vs. $\mathrm{PCI}=0.19 \sim 0.31$ in vegetative state. Every subject's consciousness index PCI combined test and algorithm steps with (1) TMS bitting, (2) HD-EEG probing of $150 \mathrm{~ms}$ in Vpp less than $5 \mu \mathrm{V}$, (3) superposition of 150 trials of evoked potentials, (4) cortical currents by means of a weighted min. norm inverse solution, (5) to extract significant sources SS(x,t), (6) sorting from bottom to top, (7) to compute Lempel-Ziv complexity, (8) normalized by source entropy.

\section{Brain Health Rule}

In Koyanaki's Lab on 31 January 2007 at Tohuku University in Japan, the first author proposed a practical signature algorithm Lock-Key Ratio for depression sub-healthstate scoring. The LKR in formula of $\mathrm{LKn} / \mathrm{LKp}$ is one kind of modified Lempel-Ziv compression algorithm with bi-threshold values of multi-fractal mean $\pm \mu$. Based on Quiet Mind's Balance Criterion $\mathrm{LKn} / \mathrm{LKp}=1$ with the tandem methods of modified LZ, multi-fractal and db3 wavelet (sub-2, $j=1,10$ ) for data in band of $0.1 \mathrm{~Hz} \sim 40 \mathrm{~Hz}$ embedded into our instrument named auricular point near infrared spectroscopy, one male master student burdened slight depressive disorder in 10subject had been successfully forecast $(\mathrm{LKn} / \mathrm{LKp}=6 / 8)$ and further verified by the psychotherapist in Hamilton depression questionnaire. Through combining the auricular point brain mapping theory with the low frequency oxygenic hemodynamics, the in vivo biomeasurement keys aim at the brain neurotransmitters' electrochemistry features of supper slow spontaneous oscillations and nonlinear time-space couplings [6].

The high detection validity of total interaction between the brain neurotransmitters had been confirmed in contrast tests of the mobile phone radiation and of the medical Calcium ion inducement.

\section{EVOLUTION RULES OF SSI}

The SSI (silent speech interface) is a system representing speech production process with unaudible acoustic signals captured from the articulators, neural or neurovascular pathways, or the brain itself of the master in BCI (brain computer interface) cycle, for enhancing masters' life qualities even if they unable to talk or need exchange information silently. Next on SSI had a short history, information 3channels and fused spider chart of 8-sensors evolution.

\section{A. A Short History}

The early idea of silent communication backed to a lipreading computer "HAL 9000" in 1968 in Stanley Kubrick science-fiction film "2001-A Space Odyssey” [6]. In 2001 Nature was in from thoughts to actions, from 2004 had touched reading regions into brain with implanted array electrodes, and the interesting neuro-decoding and imaging future trends are pointing to NIRS-based SSI started in 2005 $[7,8]$.

1) Channels of SSI: Three blocks of SSI includes information source (brain-i), and infor. sink (brain-j), handed by silent communication channels fell in essence into three categories [1-2, 7-9]: (1) traditional articulators: concerning muscle micro-vibrations of speech, located at such as voice tract, tongue, jaw and lips, or other fixed point on brain-i's face; (2) intrinsic brain: focusing on speech production areas such as Broca' area and primer motor regions for speaking; (3) neurovascular pathways: this work puts highlight onto earbrain reflection map under Chinese standard GB/T 137342008, with a new NIRS-based SSI in high recognition rate high resolution for silent communication.

2) Axes 8-sensors spider chart of SSIs: Key issues of optimized decoding validity are voiced tissue-elements or areas of brain may be monitored and what kinds of sensors will be selected?

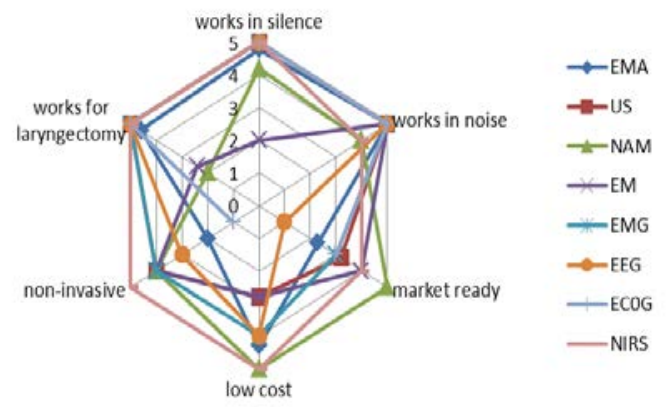

FIGURE II. 6-AXES-8-SENSORS-SPIDER CHART OF SSIS ILLUSTRATES EIGHT KINDS OF SENSORS HAD BEEN ATTEMPTED FOR DECODING RAW DATA RESULTING MAINLY FROM IMAGINED WORDS, CORRESPONDING TO ABOVE THREE CHANNEL CATEGORIES OF SSIS $[7,8,9]$.

(1) EMA (Electromagnetic articulography) sensors are used to capture the movement of fixed points on the 
articulators. Key results are for 9 words and 13 phonemes with recognition rate (RR) of over 90\% (Levinson, 2005); (2) US: to utilize ultrasound for real-time characterization of the vocal tract, imaging the tongue and lips with about 60\% RR (Denby and Stone, 2004); (3) NAM (Non-audible murmur) microphone captured digital transformation of signals nearby the neck muscles (Nakajima et al., 2003). High RR was made via improved impedance matching between the microphone diaphragm and the skin; (4) EM: to analyze glottal activity using electromagnetic or vibration sensors for speech enhancement with gains of up to $20 \mathrm{~dB}$ reported (Titze et al., 2000); (5) Surface electromyography (sEMG) probed signals from the articulator muscles or the larynx. The famous case sampled signals from three EMG sensors mounted on the speaker's face to recognize 5 Japanese vowels with $71 \% \mathrm{RR}$, and output them to a loudspeaker in real-time (Sugie and Tsunoda, 1985). Other case was in 100-word vocabulary with up to $90 \%$ RR in a speaker dependent setup (Schultz and Wand in 2009); (6) Electro-encephalographic (EEG) sensor was the BCI pioneer in 1973 by Dr. J. J. Vidal. Till 1997 Suppes et al. were the first to show that isolated spoken words can be recognized based on EEG and MEG (Magnetoencephalography) recordings. In 2006, Wester and Schultz directly recognize "unspoken speech" by EEG signals at a promising RR $35.5 \%$ under 5 words with 8 channels; (7) ECoG (Electrocorticogram) or implanted micro-electrodearrays: for interpretation of signals from implants in the speech motor cortex, early attempts focused primarily on the discrete prediction of individual phonemes based on the ensemble activity of a population of neural units (Miller et al., 2007). In 2004, Kennedy with ECoG 56-channel probing Broca's area and speech motor cortex, recognized three vowels of [hot], [heat], and [hoot] in average RR of 70\%; (8) NIRS (nearinfrared spectroscopy): Naito et al. (2007) developed a "yes" or "no" communication system for ALS (amyotrophic lateral sclerosis) locked-in patients by measuring hemodynamic responses in the brain using NIRS. However, over 30s (including rest intervals) were required for a trial. Also, the control scheme applied in test has no correlation with normal communication methods, using tasks like mental calculation, number counting, etc. In 2012, Herff based on fNIRS-BCI in test length of $8 \mathrm{~s}$, with average RRs of $71 \%$ (voiced), $61 \%$ (murmuring), and 46\% (imagined). The shortest time cycle had reported $50 \mathrm{~ms} \sim 100 \mathrm{~ms}$ for NIRS-BCI in literatures (total IEEE Journal papers on BCIs are increased about 30 times from 2003 to 2014).

All in all, the largest area of spiderweb plots may be picked up NIRS-based SSI. Next case study will report the detail of my team works in my new techs.

\section{MiLliseCOND-RESOlVED NIRS-BASED SSI}

We outline the pattern recognition flow chart as Herringbone in six letters or blocks: Objective, Data, Feature, Test, Training, and Action. Mostly from objective to action shapes in measurement-controlling- or imaging-type. Following cognitive task is to identify Chinese vowel phonemes [a], [o], and [i] based on NIRS in single-trial test at left auricular [e]-pointshown in Figure III [3,8]. Step-1: Objective definition. Chinese vowel phonemes [a], [o], and [i] recognition with NIRS-based SSI in feature of narrow-band voiceprint;

S-2: Data sampling. Key parameters are with: one pair of 875nm NIR LEDs attached two sides of front-lobe point on left ear lobe, the synchronous modulated frequency is less than $100 \mathrm{kHz}$ in pulse waveform, 1000-dot data in 1s time length. All data are captured by self-made probe-kit and Agilent 54624A storage oscilloscope in Excel 54600 Toolbar; S-3: Feature selection. Based on narrow-band spectrogram, frame length Winsiz is set 128 , and Shift=32, then quasi-formants arise in high-gamma regions. In MATLAB 7.0 instruction set two orders of $\mathrm{z}=\mathrm{z} .{ }^{*} \operatorname{conj}(\mathrm{z})$ and $\mathrm{z}=10 * \log 10(\mathrm{z})$ are the most important instructions for phonemeprints identification, the former represents scattering coefficient phase responding in complex product and the later satisfied tissue optics constrains; S-4: Feature training. For two subjectives (mail, 28 and 48 years old) the training group data are gained 10 times. Then to calculate the features mapping only phoneme; S-5: Feature testing. As S-4 more other groups are also captured for verification in parameter named RR;

S-6: Decoding rate. Table 1 shows two formants (I and II in unit $\mathrm{Hz}$ ) for three vowels in Subjective 1 and S2 with average RR near but not more than $80 \%$. From Table I we may dig out the similarities (in low, middle and high frequency for the first formants) between auricular point NIRS quasi-formants and audio voice formants in millisecond resolution.

TABLE I: TWO FORMANTS RESULTS FOR THREE VOWELS IMAGINED

\begin{tabular}{|l|c|c|l|}
\hline$/ \mathbf{H z}$ & [a] & [o] & \multicolumn{1}{|c|}{ [i] } \\
\hline S1-I & $165 \sim 195$ & $190 \sim 219$ & $445 \sim 470$ \\
\hline S1-II & $415 \sim 430$ & $360 \sim 375$ & $95 \sim 110$ \\
\hline S2-I & $255 \sim 270$ & $120 \sim 135$ & $440 \sim 500$ \\
\hline S2-II & $360 \sim 375$ & $340 \sim 350$ & $110 \sim 125$ \\
\hline
\end{tabular}

\section{CONCLUSIONS}

First perspective of figure of merits on consciousness assessment are grasped in basic three indexes. Silent speech interfaces (SSI) will enhance brain-computer communication performances under imagined or thinking cognitive state. The research methodology must match basic pattern recognition herringbone chart $(\mathrm{O}+\mathrm{D}+\mathrm{F}+2 \mathrm{~T}+\mathrm{A})$, and will trend into portable NIRS-based brain-brain communication SSI on balance of high decoding rates and low power needs. The reported briefly novel NIRS-based SSI experiment be with merits of millisecond resolution, decoding rate far more than stochastic guesses, and as analysis base of nonlinear energy operators with idea of imagined Open Sesame in 2005. By optimized techs such as the synchronous modulated NIR sensors and voiceprint, the scattering coefficients responding to neurovascular coupling under imagined vowels at front-lobe otopoint on left ear-lobe are extracted into the unique signatures, the above decoding validities were also improved by modified TEO (Teager energy operator) sign primely in our team works. 


\section{ACKNOWLEDGMENTS}

This work was supported by the National Natural Science Foundation, P. R. China in 2011 (Grant No. 61076102) and supported by the Natural Science Foundation of Jiangsu Province, P. R. China in 2014 (Grant No. BK20141196).

\section{REFERENCES}

[1] Greengard P., The neurobiology of slow synaptic transmission. Science, 294, pp. 1024-1029, 2001.

[2] Hahnloser R. H. R., Sarpeshkar R, \& Mahowald M. A., et al., Digital selection and analogue amplification coexist in a cortex-inspired silicon circuit. Nature, 405, pp. 947-951, 2000.

[3] Whenshi Li, Lie detection novel method based on brain neurotransmitter. Chinese Patent, No. 200510095198.7.

[4] Libin Hu, Wenshi Li, Research on FoM of SAR ADC. Lecture Notes in Electrical Engineering, 99, pp. 1015-1022, 2011.

[5] Talan J., A new tool for determining levels of consciousness. Neurology Today, pp. 32-33, 37, Sep.19, 2013.

[6] Denby B., Schultz T., \& Honda K., et al, Silent speech interfaces. Speech Communication, 52(4), pp.270-287, 2010.

[7] Xiaomei Pei, Jeremy Hill, Gerwin Schalk, Silent Communication. IEEE Pulse, (1), pp. 43-46, 2012.

[8] Wenshi Li, Chongyang Qian, \& Lei Li, et al, Let ear speak: an auricular frontal-lobe-point's near-infrared spectroscope brain-computer interface. Journal of Nanjing University (Natural Sciences), 48(5), pp. 654-660, 2012.

[9] Rolls E. T., Treves A., The neural encoding of information in the brain. Progress in Neurobiology, 95, pp. 448-490, 2011. 